\title{
Analysis of a stochastic distributed delay epidemic model with relapse and Gamma distribution kernel
}

\author{
Tomás Caraballo ${ }^{\mathrm{a}, *}$, Mohamed El Fatini ${ }^{\mathrm{b}}$, Mohamed El Khalifi ${ }^{\mathrm{b}}$, Richard \\ Gerlach $^{\mathrm{c}}$, Roger Pettersson ${ }^{\mathrm{d}}$ \\ ${ }^{a}$ Dpto. Ecuaciones Diferenciales y Análisis Numérico, Facultad de Matemáticas, \\ Universidad de Sevilla, Tarfia, s/n, 41012, Sevilla, Spain \\ ${ }^{b}$ Dept. of Mathematics, Faculty of Sciences, BP 133, Ibn Tofail University, Kénitra, \\ Morocco \\ ${ }^{c}$ Discipline of Business Analytics, Business School, The University of Sydney, Australia \\ ${ }^{d}$ Linnaeus University, Department of Mathematics, Växjö 351 95, Sweden
}

\begin{abstract}
In this work, we investigate a stochastic epidemic model with relapse and distributed delay. First, we prove that our model possesses and unique global positive solution. Next, by means of the Lyapunov method, we determine some sufficient criteria for the extinction of the disease and its persistence. In addition, we establish the existence of a unique stationary distribution to our model. Finally, we provide some numerical simulations for the stochastic model to assist and show the applicability and efficiency of our results.

Keywords: Epidemic model, Distributed delay, Extinction, Persistence, Stationary distribution.
\end{abstract}

\section{Introduction}

Statistics reported by the World Health Organisation shows the threats of infectious diseases to global health. It has estimated that tuberculosis caused 1.18 millions of deaths and 2.56 millions of peoples died from pneumonia in 2017. At the end of 2018, a total of 32 millions of peoples were killed by HIV since the beginning of the epidemic. Biological literature has been enriched by

\footnotetext{
* Corresponding author

Email address: caraball@us.es (Tomás Caraballo)
} 
contribution of mathematicians to understand, predict behaviors and control the spread of detrimental epidemics. Since the basic SIR model introduced in works of Kermack and McKendrick [13, 14, 15, many important extensions have been developed including different characteristics of the epidemics [1, 2, [3, 4, 17, 24, 26, 12, 20, 28, 8, 18. Since many species should reveal time delay, the introduction of time delays into compartmental epidemic models presents a fascinating improvement as it models sojourn times in a specified state, e.g. the infective state, see [21, 23, 27, 5]. In [19], authors studied the following stochastic SIR epidemic model with distributed time delay

$$
\left\{\begin{aligned}
d S(t) & =\left(\mu-\mu S(t)-\beta S(t) \int_{-\infty}^{t} D(t-s) I(s) d s\right) d t+\sigma S d B(t) \\
d I(t) & =\left(\beta S(t) \int_{-\infty}^{t} D(t-s) I(s) d s-(\mu+\lambda+\delta) I(t)\right) d t \\
d R(t) & =(\lambda I(t)-\mu R(t)) d t
\end{aligned}\right.
$$

where $S(t), I(t)$ and $R(t)$ represent the densities of susceptible, infected and recovered individuals at time $t$ respectively. The parameter $\mu$ is the recruitment rate, $\beta$ the infection transmission rate, $\lambda$ the recovery rate and $\delta$ the death rate revealed by the disease. The quantity $\int_{-\infty}^{t} D(t-s) I(s) d s$ is the force of infection at time $t$. The function $D:[0, \infty) \rightarrow[0, \infty)$, called delay kernel, is an $L^{1}$-function verifying $\int_{0}^{\infty} D(s) d s=1$. The standard Brownian motion $B(t)$ with intensity $\sigma$ is used to model the environmental noise on susceptible class. Connected with the above statements, we suggest to study the dynamics of a stochastic SIR epidemic model incorporating relapse, possessing distributed time delay with environmental noise proportional to $S(t), I(t)$ and $R(t)$ as

$$
\left\{\begin{aligned}
d S(t)= & \left(\mu-\mu S(t)-\beta S(t) \int_{-\infty}^{t} D(t-s) I(s) d s\right) d t+\sigma_{1} S(t) d B_{1}(t) \\
d I(t)= & \left(\beta S(t) \int_{-\infty}^{t} D(t-s) I(s) d s-(\mu+\lambda+\delta) I(t)+\gamma R(t)\right) d t \\
& +\sigma_{2} I(t) d B_{2}(t) \\
d R(t)= & (\lambda I(t)-(\mu+\gamma) R(t)) d t+\sigma_{3} R(t) d B_{3}(t)
\end{aligned}\right.
$$

where $\gamma$ is the rate of reinfection, and $B_{i}(t), i=1,2,3$ are independent Brownian motions with intensities $\sigma_{i}, i=1,2,3$.

In practice, it is convenient to use the kernel delay $D$ with Gamma distribution 
[22], that is

$$
D(s)=\frac{s^{n} \alpha^{n+1} e^{-\alpha s}}{n !}, \quad s>0,
$$

where the positive number $\alpha$ is the rate of decay of effect of past memories. In this paper, we consider the weak kernel (i.e. $n=1$ ) and for simplicity we let

$$
Z(t)=\int_{-\infty}^{t} \alpha e^{-\alpha(t-s)} I(s) d s,
$$

then it follows that

$$
d Z(t)=\alpha(I(t)-Z(t)) d t
$$

We also allow the component $Z(t)$ to be affected by the environmental variability as it depends of the perturbed density of infected individuals. Hence, system (2) can be rewritten as

$$
\left\{\begin{aligned}
d S(t) & =(\mu-\mu S(t)-\beta S(t) Z(t)) d t+\sigma_{1} S(t) d B_{1}(t) \\
d I(t) & =(\beta S(t) Z(t)-(\mu+\lambda+\delta) I(t)+\gamma R(t)) d t+\sigma_{2} I(t) d B_{2}(t) \\
d R(t) & =(\lambda I(t)-(\mu+\gamma) R(t)) d t+\sigma_{3} R(t) d B_{3}(t) \\
d Z(t) & =\alpha(I(t)-Z(t)) d t+\sigma_{4} Z(t) d B_{4}(t)
\end{aligned}\right.
$$

where $B_{4}(t)$ stands for Brownian motion with intensity $\sigma_{4}$.

Despite the realism presented by epidemic models with distributed delay, their stochastic analysis is still quite difficult and limited. To the best of our knowledge, only sufficient conditions are obtained for the extinction and persistence of the diseases with such kind of delay (see, for instance, (1)). Actually, more general results are proved for models with maturation and bounded delays [9, 25].

The content of this paper is structured as follows. Section 2 is devoted to establish the existence of a unique global positive solution for the stochastic epidemic model (3). In Section 3, by means of Lyapunov functions, we establish a sufficient criterion for the exponential extinction of the disease. The persistence in mean result under some appropriate conditions is proved in Section 4. In Section 5, we analyze the existence of an ergodic stationary distribution to system (3). Finally, in Section 6, some numerical examples are presented to 
provide a practical exhibition and explanation to our analytical results.

Throughout this paper, let $\left(\Omega, \mathcal{F},\left\{\mathcal{F}_{t}\right\}_{t \geq 0}, \mathbb{P}\right)$ be a complete probability space with a filtration $\left\{\mathcal{F}_{t}\right\}_{t \geq 0}$ satisfying usual conditions. Define the space $\mathbb{R}_{+}^{n}$ as follows

$$
\mathbb{R}_{+}^{n}=\left\{x \in \mathbb{R}^{n}: x_{i}>0, i=1, \cdots, n\right\} .
$$

Let $X_{t}$ be a regular homogeneous Markov process in $\mathbb{R}^{n}$ described by the SDE

$$
d X_{t}=f(X) d t+\sum_{r=1}^{k} g_{r}(X) d B_{r}(t), \quad X(0)=X_{0},
$$

with the diffusion matrix defined by

$$
A(x)=\left(a_{i j}(x)\right), \quad a_{i j}(x)=\sum_{r=1}^{k} g_{r}^{i}(x) g_{r}^{j}(x) .
$$

We introduce the differential operator $\mathcal{L}$ associated to (4) and acts on any twice continuously differentiable function $V$ as follows

$$
\mathcal{L} V(x)=\sum_{i=1}^{n} f_{i}(x) \frac{\partial V(x)}{\partial x_{i}}+\frac{1}{2} \sum_{i, j=1}^{n} \frac{\partial^{2} V(x)}{\partial x_{i} \partial x_{j}} .
$$

The following lemma is a worthwhile criterion for the existence of ergodic stationary distributions [16].

Lemma 1. Assume the following assumptions hold,

1. There exists a positive number $\kappa$ such that $\sum_{i, j=1}^{n} a_{i j} \xi_{i} \xi_{j} \geq \kappa|\xi|^{2}, \xi \in D$, where $D \subset \mathbb{R}^{n}$ is a bounded open set with compact closure.

2. There exists a nonnegative $\mathcal{C}^{2}$-function $V: D^{c} \rightarrow \mathbb{R}$ such that $\mathcal{L} V$ is negative for any $x \in D^{c}=\mathbb{R}^{n} \backslash D$.

Then, the Markov process $X(t)$ has a unique ergodic stationary distribution $\mu(\cdot)$ with density in $\mathbb{R}^{n}$ such that

$$
\mathbb{P}\left\{\lim _{T \rightarrow \infty} \frac{1}{T} \int_{0}^{T} h(X(t)) d t=\int_{\mathbb{R}^{n}} h(x) \mu(d x)\right\}=1,
$$

for any $x \in \mathbb{R}^{n}$ and $h(\cdot)$ is an integrable function with respect to the measure $\mu$. 


\section{Existence and uniqueness of global positive solution}

In the sequel, we show that system (3) admits a unique global nonnegative solution for any initial condition on $\mathbb{R}_{+}^{4}$.

Theorem 2.1. For any given initial value $(S(0), I(0), R(0), Z(0)) \in \mathbb{R}_{+}^{4}$, there exists a unique local solution $(S(t), I(t), R(t), Z(t))$ to system (3) on $t \geq 0$ and the solution remains in $\mathbb{R}_{+}^{4}$ almost surely.

Proof. Let $(S(0), I(0), R(0), Z(0)) \in \mathbb{R}_{+}^{4}$. Since the considered system has a locally Lipschitz coefficients of linear growth, there exists a unique solution $(S(t), I(t), R(t))$ on $t \in\left[0, \tau_{e}\right)$, where $\tau_{e}$ is the explosion time. Now, we shall prove that $\tau_{e}=\infty$ a.s. To this end, we need to construct a $\mathcal{C}^{2}$-function $V$ : $\mathbb{R}_{+}^{4} \mapsto \mathbb{R}_{+} \cup\{0\}$ such that

$$
\liminf _{k \rightarrow \infty,(S, I, R, Z) \in \mathbb{R}_{+}^{4} \backslash D_{k}} V(S, I, R, Z)=\infty \quad \text { and } \quad \mathcal{L} V(S, I, R, Z) \leq C,
$$

where $D_{k}=\left(\frac{1}{k}, k\right)^{4}$ for $k \geq k_{0} \geq 1, k_{0}$ is a sufficiently large integer such that $(S(0), I(0), R(0), Z(0)) \in D_{k_{0}}$ and $C$ is a positive constant.

For any $k \geq k_{0}$, we define $\tau_{k}=\inf \left\{t \in\left[0, \tau_{e}\right):(S, I, R, Z) \notin D_{k}\right\}$. Obviously, $\left(\tau_{k}\right)_{k \geq k_{0}}$ is an increasing sequence with limit $\tau_{\infty}$ verifying $\tau_{\infty} \leq \tau_{e}$ a.s. It is sufficient to prove that $\tau_{\infty}=\infty$ a.s. If this is false, then there is a pair of constants $T>0$ and $\varepsilon \in(0,1)$ such that $\mathbb{P}\left\{\tau_{\infty} \leq T\right\}>\varepsilon$. Hence, there is an integer $k_{1} \geq k_{0}$ such that $\mathbb{P}\left\{\tau_{k} \leq T\right\} \geq \varepsilon$ for all $k \geq k_{1}$.

Let $a$ and $b$ be two positive constants and define

$V(S, I, R, Z)=\left(S-a-a \ln \frac{S}{a}\right)+(I-1-\ln I)+(R-1-\ln R)+b\left(Z-b-b \ln \frac{Z}{b}\right)$.

Since $-\ln x \underset{x \rightarrow 0^{+}}{\longrightarrow} \infty$ and $-x-m-m \ln \frac{x}{m} \underset{x \rightarrow \infty}{\longrightarrow} \infty$ for any $m>0$, one can easily obtain that

$$
\liminf _{k \rightarrow \infty,(S, I, R, Z) \in \mathbb{R}_{+}^{4} \backslash D_{k}} V(S, I, R, Z)=\infty .
$$


Now, using Itô's formula on $V$, we obtain that

$$
\begin{aligned}
d V(S, I, R, Z)= & \mathcal{L} V(S, I, R, Z) d t+\left(1-\frac{a}{S}\right) \sigma_{1} S d B_{1}+\left(1-\frac{1}{I}\right) \sigma_{2} I d B_{2} \\
& +\left(1-\frac{1}{R}\right) \sigma_{3} R d B_{3}+\left(b-\frac{b^{2}}{Z}\right) \sigma_{4} Z d B_{4}
\end{aligned}
$$

where

$$
\begin{aligned}
\mathcal{L} V(S, I, R, Z)= & \left(1-\frac{a}{S}\right)(\mu-\mu S-\beta S Z)+\left(1-\frac{1}{I}\right)(\beta S Z-(\mu+\lambda+\delta) I+\gamma R) \\
& +\left(1-\frac{1}{R}\right)(\lambda I-(\mu+\gamma) R)+\left(b-\frac{b^{2}}{Z}\right)(\alpha I-\alpha Z)+\frac{a \sigma_{1}^{2}}{2} \\
& +\frac{\sigma_{2}^{2}}{2}+\frac{\sigma_{3}^{2}}{2}+\frac{b \sigma_{4}^{2}}{2} \\
= & 3 \mu+a \mu+\lambda+\delta+\gamma+\frac{a \sigma_{1}^{2}}{2}+\frac{\sigma_{2}^{2}}{2}+\frac{\sigma_{3}^{2}}{2}+\frac{b \sigma_{4}^{2}}{2}+(b \alpha-(\mu+\delta)) I \\
& +(a \beta-b \alpha) Z-\left(\mu\left(S+R+\frac{a}{S}\right)+\frac{\beta S Z}{I}+\frac{\gamma R}{I}+\frac{\lambda I}{R}+\frac{b^{2} \alpha I}{Z}\right) .
\end{aligned}
$$

Choosing $a=\frac{\mu+\delta}{\beta}$ and $b=\frac{\mu+\delta}{\alpha}$ leads to

$$
\mathcal{L} V(S, I, R, Z) \leq C,
$$

where $C=3 \mu+a \mu+\lambda+\delta+\gamma+\frac{a \sigma_{1}^{2}}{2}+\frac{\sigma_{2}^{2}}{2}+\frac{\sigma_{3}^{2}}{2}+\frac{b \sigma_{4}^{2}}{2}$.

The remaining part of the proof is similar to Theorem 1.2 of [6]. However, for the convenience of the reader, we will include it here.

By (5), we have

$$
\begin{aligned}
d V(S, I, R, Z) \leq & C d t+\left(1-\frac{a}{S}\right) \sigma_{1} S d B_{1}+\left(1-\frac{1}{I}\right) \sigma_{2} I d B_{2} \\
& +\left(1-\frac{1}{R}\right) \sigma_{3} R d B_{3}+\left(b-\frac{b^{2}}{Z}\right) \sigma_{4} Z d B_{4} .
\end{aligned}
$$

Integrating both sides of the last inequality over $\left[0, \tau_{k} \wedge T\right]$ yields

$$
\begin{gathered}
\mathbb{E} V\left(S\left(\tau_{k} \wedge T\right), I\left(\tau_{k} \wedge T\right), R\left(\tau_{k} \wedge T\right), Z\left(\tau_{k} \wedge T\right)\right) \\
\leq V(S(0), I(0), R(0), Z(0))+C T,
\end{gathered}
$$


which gives that

$$
\begin{aligned}
V(S(0), & I(0), R(0), Z(0))+C T \\
& \left.\geq \mathbb{E}\left[1_{\{} \tau_{k} \leq T\right\} V\left(S\left(\tau_{k} \wedge T\right), I\left(\tau_{k} \wedge T\right), R\left(\tau_{k} \wedge T\right), Z\left(\tau_{k} \wedge T\right)\right)\right] \\
& \geq \varepsilon \theta_{k},
\end{aligned}
$$

where

$$
\begin{aligned}
\theta_{k}= & \left(k-a-a \ln \frac{k}{a}\right) \wedge\left(\frac{1}{k}-a+a \ln (k a)\right) \wedge(k-1-\ln k) \\
& \wedge\left(\frac{1}{k}-1+\ln k\right) \wedge b\left(k-b-b \ln \frac{k}{b}\right) \wedge b\left(\frac{1}{k}-b+b \ln (k b)\right) .
\end{aligned}
$$

Letting $k \rightarrow \infty$ leads to the contradiction $\infty>V(S(0), I(0), R(0), Z(0))+C T=$ $\infty$. Hence $\tau_{\infty}=\infty$ a.s. This concludes the proof.

\section{Extinction of the disease}

In this section, we establish sufficient conditions for the extinction of the disease in our model (3). We set the parameter

$$
\mathcal{R}_{0}=\frac{\beta}{\mu+\lambda+\delta}+\frac{\lambda \gamma}{(\mu+\lambda+\delta)(\mu+\gamma)} .
$$

Then, we have the following theorem.

Theorem 3.1. Let $(S(t), I(t), R(t), Z(t))$ be the solution to system (3) for an initial value $(S(0), I(0), R(0), Z(0))$ in $\mathbb{R}_{+}^{4}$. Assume that

$$
\begin{gathered}
\mathcal{R}_{0}<1, \quad \sigma_{1}^{2} \leq 2 \mu \quad \text { and } \\
\min \{\mu+\lambda+\delta, \mu+\gamma, \alpha\}\left(\sqrt{\mathcal{R}_{0}}-1\right)+\frac{\beta \alpha \sigma_{1} \sqrt{\mathcal{R}_{0}}}{(\mu+\lambda+\delta) \sqrt{2 \mu-\sigma_{1}^{2}}}<0
\end{gathered}
$$

Then, the disease dies out exponentially with probability one. Moreover, the distribution of $S(t)$ converges weakly to the measure which has the density

$$
p(x)=Q x^{-\frac{2 \mu}{\sigma_{1}^{2}}-2} e^{-\frac{2 \mu}{\sigma_{1}^{2} x}}, \quad x>0,
$$

$20 \quad$ where the normalisation constant $Q=\left(\frac{2 \mu}{\sigma_{1}^{2}}\right)^{\frac{2 \mu}{\sigma_{1}^{2}}+1}\left(\Gamma\left(\frac{2 \mu}{\sigma_{1}^{2}}+1\right)\right)^{-1}$. 
Proof. First, we consider $X_{t}$ solution to the following SDE

$$
d X_{t}=(\mu-\mu X) d t+\sigma_{1} X d B_{1}(t), \quad X(0)=S(0)>0 .
$$

Let $q(x)=\exp \left(-2 \int_{1}^{x} \frac{\mu-\mu u}{\left(\sigma_{1} u\right)^{2}} d u\right)$. We have

$$
q(x)=\exp \left(-2 \frac{\mu}{\sigma_{1}^{2}}\left(1-\ln x-\frac{1}{x}\right)\right)=e^{-\frac{2 \mu}{\sigma_{1}^{2}}} x^{\frac{2 \mu}{\sigma_{1}^{2}}} e^{\frac{2 \mu}{\sigma_{1}^{2} x}} .
$$

Hence,

$$
\int_{1}^{\infty} q(x) d x=\infty, \int_{0}^{1} q(x) d x=\infty \quad \text { and } \quad \int_{0}^{\infty} \frac{1}{\sigma_{1}^{2}} x^{-2} q(x)^{-1} d x<\infty .
$$

Therefore, by Theorem 1.16 in [17, (6) has the ergodic property and the density of its invariant law is given by

$$
p(x)=Q x^{-\frac{2 \mu}{\sigma_{1}^{2}}-2} e^{-\frac{2 \mu}{\sigma_{1}^{2} x}},
$$

where $Q=\left(\frac{2 \mu}{\sigma_{1}^{2}}\right)^{\frac{2 \mu}{\sigma_{1}^{2}}+1}\left(\Gamma\left(\frac{2 \mu}{\sigma_{1}^{2}}+1\right)\right)^{-1}$. By the comparison theorem, we have $S(t) \leq X(t), t \geq 0$ a.s. By direct computations, one can obtain

$$
\begin{aligned}
\int_{0}^{\infty} x p(x) d x & =Q \int_{0}^{\infty} x^{-\frac{2 \mu}{\sigma_{1}^{2}}-1} e^{-\frac{2 \mu}{\sigma_{1}^{2} x}} d x \\
& =Q\left(\frac{2 \mu}{\sigma_{1}^{2}}\right)^{-\frac{2 \mu}{\sigma_{1}^{2}}} \Gamma\left(\frac{2 \mu}{\sigma_{1}^{2}}\right) \\
& =1
\end{aligned}
$$

and

$$
\begin{aligned}
\int_{0}^{\infty} x^{2} p(x) d x & =Q \int_{0}^{\infty} x^{-\frac{2 \mu}{\sigma_{1}^{2}}} e^{-\frac{2 \mu}{\sigma_{1}^{2} x}} d x \\
& =Q\left(\frac{2 \mu}{\sigma_{1}^{2}}\right)^{-\frac{2 \mu}{\sigma_{1}^{2}}+1} \Gamma\left(\frac{2 \mu}{\sigma_{1}^{2}}-1\right) \\
& =\frac{2 \mu}{2 \mu-\sigma_{1}^{2}} .
\end{aligned}
$$

Then,

$$
\int_{0}^{\infty}(x-1)^{2} p(x) d x=\int_{0}^{\infty}\left(x^{2}-2 x+1\right) p(x) d x=\frac{\sigma_{1}^{2}}{2 \mu-\sigma_{1}^{2}} .
$$


Next, we consider the nonnegative $\mathcal{C}^{2}$-function $V_{1}(I, R, Z)=\alpha_{1} I+\alpha_{2} R+\alpha_{3} Z$, where $\alpha_{1}=\frac{\omega_{1}}{\mu+\lambda+\delta}, \alpha_{2}=\frac{\omega_{2}}{\mu+\gamma}$, and $\alpha_{3}=\frac{\omega_{3}}{\alpha} \cdot \omega_{i}, i=1, \cdots, 3$ are positive constants to be determined. Using Itô's formula for $\ln V_{1}$, we have

$$
\begin{aligned}
d \ln V_{1}= & \frac{1}{V_{1}}\left\{\alpha_{1}(\beta S Z-(\mu+\lambda+\delta) I+\gamma R)+\alpha_{2}(\lambda I-(\mu+\gamma) R)+\alpha_{3} \alpha(I-Z)\right\} d t \\
& -\frac{1}{2 V_{1}^{2}}\left(\left(\alpha_{1} \sigma_{2} I\right)^{2}+\left(\alpha_{2} \sigma_{3} R\right)^{2}+\left(\alpha_{3} \sigma_{4} Z\right)^{2}\right) d t \\
& +\frac{1}{V_{1}}\left(\sigma_{2} I d B_{2}+\sigma_{3} R d B_{3}+\sigma_{4} Z d B_{4}\right) \\
= & \mathcal{L} \ln V_{1} d t+\frac{1}{V}\left(\sigma_{2} I d B_{2}+\sigma_{3} R d B_{3}+\sigma_{4} Z d B_{4}\right),
\end{aligned}
$$

where

$$
\begin{aligned}
\mathcal{L} \ln V_{1}= & \frac{1}{V_{1}}\left\{\alpha_{1}(\beta Z-(\mu+\lambda+\delta) I+\gamma R)+\alpha_{2}(\lambda I-(\mu+\gamma) R)+\alpha_{3} \alpha(I-Z)\right\} \\
& +\frac{\alpha_{1} \beta}{V_{1}}(S-1) Z-\frac{1}{2 V^{2}}\left(\left(\alpha_{1} \sigma_{2} I\right)^{2}+\left(\alpha_{2} \sigma_{3} R\right)^{2}+\left(\alpha_{3} \sigma_{4} Z\right)^{2}\right) \\
\leq & \frac{\alpha_{1} \beta}{\alpha_{3}}|X-1| \\
& +\frac{1}{V_{1}}\left\{\alpha_{1}(\beta Z-(\mu+\lambda+\delta) I+\gamma R)+\alpha_{2}(\lambda I-(\mu+\gamma) R)+\alpha_{3} \alpha(I-Z)\right\} \\
\leq & \frac{\alpha_{1} \beta}{\alpha_{3}}|X-1|+\frac{1}{V_{1}}\left\{\frac{\omega_{1}}{\mu+\lambda+\delta}(\beta Z-(\mu+\lambda+\delta) I+\gamma R)\right. \\
& \left.+\frac{\omega_{2}}{\mu+\gamma}(\lambda I-(\mu+\gamma) R)+\omega_{3} \alpha(I-Z)\right\} .
\end{aligned}
$$

Let $M_{0}$ be the matrix defined by

$$
M_{0}=\left(\begin{array}{ccc}
0 & \frac{\gamma}{\mu+\lambda+\delta} & \frac{\beta}{\mu+\lambda+\delta} \\
\frac{\lambda}{\mu+\gamma} & 0 & 0 \\
1 & 0 & 0
\end{array}\right),
$$

with eigenvalue $\sqrt{\mathcal{R}_{0}}=\sqrt{\frac{\beta}{\mu+\lambda+\delta}+\frac{\lambda \gamma}{(\mu+\lambda+\delta)(\mu+\gamma)}}$. Choosing the vector $\left(\omega_{1}, \omega_{2}, \omega_{3}\right)=$ $\left(\sqrt{\mathcal{R}_{0}}, \frac{\lambda}{\mu+\gamma}, 1\right)$ leads to

$$
\left(\omega_{1}, \omega_{2}, \omega_{3}\right) M_{0}=\sqrt{\mathcal{R}_{0}}\left(\omega_{1}, \omega_{2}, \omega_{3}\right)
$$


Therefore,

$$
\begin{aligned}
\mathcal{L} \ln V_{1} & \leq \frac{\alpha_{1} \beta}{\alpha_{3}}|X-1|+\frac{1}{V_{1}}\left(\omega_{1} \omega_{2} \omega_{3}\right)\left(M_{0}(I, R, Z)^{T}-(I, R, Z)^{T}\right) \\
& =\frac{\alpha_{1} \beta}{\alpha_{3}}|X-1|+\frac{1}{V_{1}}\left(\sqrt{\mathcal{R}_{0}}-1\right)\left(\omega_{1} I+\omega_{2} R+\omega_{3} Z\right) \\
& \leq \frac{\alpha_{1} \beta}{\alpha_{3}}|X-1|+\min \{\mu+\lambda+\delta, \mu+\gamma, \alpha\}\left(\sqrt{\mathcal{R}_{0}}-1\right) .
\end{aligned}
$$

Hence, we obtain the inequality

$$
\begin{aligned}
d \ln V_{1} \leq & \frac{\alpha_{1} \beta}{\alpha_{3}}|X-1|+\min \{\mu+\lambda+\delta, \mu+\gamma, \alpha\}\left(\sqrt{\mathcal{R}_{0}}-1\right) \\
& +\frac{1}{V}\left(\sigma_{2} I d B_{2}+\sigma_{3} R d B_{3}++\sigma_{4} Z d B_{4}\right) .
\end{aligned}
$$

Integrating both sides and dividing by $t$ gives

$$
\begin{aligned}
\frac{\ln V_{1}(I(t), R(t), Z(t))}{t} \leq & \frac{\ln V_{1}(I(0), R(0), Z(0))}{t} \\
& +\min \{\mu+\lambda+\delta, \mu+\gamma, \alpha\}\left(\sqrt{\mathcal{R}_{0}}-1\right) \\
& +\frac{\alpha_{1} \beta}{\alpha_{3}} \frac{1}{t} \int_{0}^{t}|X(s)-1| d s+\frac{N_{t}}{t}
\end{aligned}
$$

where $N_{t}=\int_{0}^{t} \frac{\sigma_{2} I}{V_{1}} d B_{2}+\int_{0}^{t} \frac{\sigma_{3} R}{V_{1}} d B_{3}+\int_{0}^{t} \frac{\sigma_{4} Z}{V_{1}} d B_{4}$ is a local martingale with finite quadratic variation. Hence, $\lim _{t \rightarrow \infty} \frac{N_{t}}{t}=0$ a.s. by the strong law of large numbers for local martingales.

As the solution to system (6) is ergodic and $\int_{0}^{\infty} x p(x) d x<\infty$, then

$$
\begin{aligned}
\lim _{t \rightarrow \infty} \frac{1}{t} \int_{0}^{t}|X(s)-1| d s=\int_{0}^{\infty}|x-1| p(x) d x & \leq\left(\int_{0}^{\infty}(x-1)^{2} p(x) d x\right)^{\frac{1}{2}} \\
& =\frac{\sigma_{1}}{\sqrt{2 \mu-\sigma_{1}^{2}}} .
\end{aligned}
$$


Consequently

$$
\begin{aligned}
& \limsup _{t \rightarrow \infty} \frac{\ln V_{1}(I(t), R(t), Z(t))}{t} \leq \min \{\mu+\lambda+\delta, \mu+\gamma, \alpha\}\left(\sqrt{\mathcal{R}_{0}}-1\right) \\
&+\frac{\alpha_{1} \beta}{\alpha_{3}} \frac{\sigma_{1}}{\sqrt{2 \mu-\sigma_{1}^{2}}} \\
&= \min \{\mu+\lambda+\delta, \mu+\gamma, \alpha\}\left(\sqrt{\mathcal{R}_{0}}-1\right) \\
&+\frac{\beta \alpha \sigma_{1} \sqrt{\mathcal{R}_{0}}}{(\mu+\lambda+\delta) \sqrt{2 \mu-\sigma_{1}^{2}}} \\
&<0 \quad \text { a.s. }
\end{aligned}
$$

which is the desired result. Moreover,

$$
\limsup _{t \rightarrow \infty} \frac{\ln I(t)}{t}<0, \quad \limsup _{t \rightarrow \infty} \frac{\ln Z(t)}{t}<0 \quad \text { a.s. }
$$

This implies that $I(t)$ goes to 0 exponentially with probability 1 . The proof is then complete.

\section{Persistence in mean of the Disease}

The study of the persistence of diseases is an important approach to know more on epidemics behaviors, since it provides the conditions under which the diseases are prevalent. For reasons of simplification, we define the quantity

$$
\mathcal{R}_{s}^{0}=\frac{\beta K}{\mu+\lambda+\delta+\frac{\sigma_{2}^{2}}{2}+\beta K \frac{\sigma_{4}^{2}}{2 \alpha}-\frac{\lambda \gamma}{\mu+\gamma+\frac{\sigma_{3}^{2}}{2}}},
$$

$25 \quad$ where $K=\frac{\mu}{\mu+\frac{\sigma_{1}^{2}}{2}}$. We then have the following result.

Theorem 4.1. If $\mathcal{R}_{s}^{0}>1$, then for any given initial value $(S(0), I(0), R(0), Z(0)) \in$ $\mathbb{R}_{+}^{4}$, the corresponding solution to $(3)$ verifies

$$
\liminf _{t \rightarrow \infty} \frac{1}{t} \int_{0}^{t} I(s) d s \geq \Lambda\left(1-\frac{1}{\mathcal{R}_{s}^{0}}\right) \quad \text { a.s. }
$$

where $\Lambda=\frac{\mu \alpha}{\beta\left(\alpha+\frac{\sigma_{4}^{2}}{2}\right)}$. In other words, the epidemic will be permanent. 
Proof. Let $(S(0), I(0), R(0), Z(0)) \in \mathbb{R}_{+}^{4}$ and consider the function

$$
V_{2}(S, I, R, Z)=-\beta_{1} \ln S-\ln I-\beta_{2} \ln R-\beta_{3} \ln Z+\beta_{4} Z,
$$

where $\beta_{i}, i=1, \cdots, 4$ are positive constants to be specified later. The function $V_{2}$ is continuous and goes to $\infty$ as $(S, I, R, Z)$ goes to the boundary of $\mathbb{R}_{+}^{4}$. Thus it must has a lower bound $M$. Then we define the nonnegative function $\tilde{V}_{2}=V_{2}(S, I, R, Z)-M$. Applying Itô's formula to $\tilde{V}_{2}$, we obtain

$$
\begin{aligned}
d \tilde{V}_{2}= & -\frac{\beta_{1}}{S}(\mu-\mu S-\beta S Z)+\beta_{1} \frac{\sigma_{1}^{2}}{2}-\frac{1}{I}(\beta S Z-(\mu+\lambda+\delta) I+\gamma R)+\frac{\sigma_{2}^{2}}{2} \\
& -\frac{\beta_{2}}{R}(\lambda I-(\mu+\gamma) R)+\beta_{2} \frac{\sigma_{3}^{2}}{2}-\beta_{3} \frac{\alpha}{Z}(I-Z)+\beta_{3} \frac{\sigma_{4}^{2}}{2}+\beta_{4} \alpha(I-Z) \\
& -\left(\beta_{1} \sigma_{1} d B_{1}+\sigma_{2} d B_{2}+\beta_{2} \sigma_{3} d B_{3}+\sigma_{4}\left(\beta_{3}-\beta_{4} Z\right) d B_{4}\right) \\
= & \mathcal{L} \tilde{V}_{2} d t-\left(\beta_{1} \sigma_{1} d B_{1}+\sigma_{2} d B_{2}+\beta_{2} \sigma_{3} d B_{3}\right),
\end{aligned}
$$

where

$$
\begin{aligned}
\mathcal{L} \tilde{V}_{2}= & -\frac{\beta S Z}{I}-\frac{\beta_{1} \mu}{S}-\frac{\beta_{3} \alpha I}{Z}-\frac{\gamma R}{I}-\frac{\beta_{2} \lambda I}{R}+\left(\beta_{1} \beta-\beta_{4} \alpha\right) Z+\beta_{1}\left(\mu+\frac{\sigma_{1}^{2}}{2}\right) \\
& +\mu+\lambda+\delta+\frac{\sigma_{2}^{2}}{2}+\beta_{2}\left(\mu+\gamma+\frac{\sigma_{3}^{2}}{2}\right)+\beta_{3}\left(\alpha+\frac{\sigma_{4}^{2}}{2}\right)+\beta_{4} \alpha I,
\end{aligned}
$$

Choosing $\beta_{4}=\beta_{1} \beta / \alpha$ and using the facts that

$$
-\frac{\beta S Z}{I}-\frac{\beta_{1} \mu}{S}-\frac{\beta_{3} \alpha I}{Z} \leq-3 \sqrt[3]{\beta \mu \alpha \beta_{1} \beta_{3}} \quad \text { and } \quad-\frac{\gamma R}{I}-\frac{\beta_{2} \lambda I}{R} \leq-2 \sqrt{\lambda \gamma \beta_{2}}
$$

we obtain

$$
\begin{aligned}
\mathcal{L} \tilde{V}_{2} \leq & -3 \sqrt[3]{\beta \mu \alpha \beta_{1} \beta_{3}}-2 \sqrt{\lambda \gamma \beta_{2}}+\beta_{1}\left(\mu+\frac{\sigma_{1}^{2}}{2}\right)+\mu+\lambda+\delta+\frac{\sigma_{2}^{2}}{2} \\
& +\beta_{2}\left(\mu+\gamma+\frac{\sigma_{3}^{2}}{2}\right)+\beta_{3}\left(\alpha+\frac{\sigma_{4}^{2}}{2}\right)+\beta_{4} \alpha I .
\end{aligned}
$$

For $\beta_{2}=\lambda \gamma /\left(\mu+\gamma+\frac{\sigma_{3}^{2}}{2}\right)^{2}, \beta_{1}=\beta K^{2}\left(\alpha+\frac{\sigma_{4}^{2}}{2}\right) /(\mu \alpha)$ and $\beta_{3}=\beta K /\left(\alpha+\frac{\sigma_{4}^{2}}{2}\right)$, we get

$$
\begin{aligned}
\mathcal{L} \tilde{V}_{2} & \leq-\beta K+\mu+\lambda+\delta+\frac{\sigma_{2}^{2}}{2}+\beta K \frac{\sigma_{4}^{2}}{2 \alpha}-\frac{\lambda \gamma}{\mu+\gamma+\frac{\sigma_{3}^{2}}{2}}+\beta_{1} \beta I \\
& =-\beta K\left(1-\frac{1}{\mathcal{R}_{s}^{0}}\right)+\beta_{1} \beta I .
\end{aligned}
$$


We further obtain

$$
\begin{aligned}
d \tilde{V}_{2} \leq & \left(-\beta K\left(1-\frac{1}{\mathcal{R}_{s}^{0}}\right)+\beta_{1} \beta I\right) d t \\
& -\left(\beta_{1} \sigma_{1} d B_{1}+\sigma_{2} d B_{2}+\beta_{2} \sigma_{3} d B_{3}+\sigma_{4}\left(\beta_{3}-\beta_{4} Z\right) d B_{4}\right) .
\end{aligned}
$$

Integrating both sides of the last inequality and dividing by $t$, leads to

$$
\begin{aligned}
0 \leq & \frac{\tilde{V}_{2}(S(t), I(t), R(t), Z(t))}{t} \\
\leq & \frac{\tilde{V}_{2}(S(0), I(0), R(0), Z(0))}{t}-\beta K\left(1-\frac{1}{\mathcal{R}_{s}^{0}}\right) \\
& +\beta_{1} \beta \frac{1}{t} \int_{0}^{t} I(s) d s+M_{t},
\end{aligned}
$$

where $M_{t}=-\frac{1}{t}\left(\beta_{1} \sigma_{1} B_{1}(t)+\sigma_{2} B_{2}(t)+\beta_{2} \sigma_{3} B_{3}(t)+\sigma_{4} \int_{0}^{t}\left(\beta_{3}-\beta_{4} Z\right) d B_{4}\right)$ is a continuous local martingale. Using the large numbers theorem for martingales, we obtain $\lim _{t \rightarrow \infty} M_{t}=0$. Therefore

$$
\liminf _{t \rightarrow \infty} \frac{1}{t} \int_{0}^{t} I(s) d s \geq \frac{K}{\beta_{1}}\left(1-\frac{1}{\mathcal{R}_{s}^{0}}\right) \quad \text { a.s. }
$$

This completes the proof.

\section{Stationary distribution and positive recurrence}

In the previous sections, we have investigated both the extinction and the permanence of the disease under some conditions. In this section, we will show that the solution to system (3) possesses a unique stationary distribution by means of the method given in [16] and used in [29].

Theorem 5.1. For any $(S(0), I(0), R(0), Z(0)) \in \mathbb{R}_{+}^{4}$, the corresponding so-

lution to system (3) is positive recurrent and has a unique ergodic stationary distribution if the condition $\mathcal{R}_{s}^{0}>1$ holds.

Proof. It is easy to verify the first condition of Lemma 1 We only have to prove that the second condition is also verified. Let us consider the $\mathcal{C}^{2}$-function $V_{6}$ defined by

$$
V_{5}(S, I, R, Z)=M V_{2}+V_{3}+V_{4},
$$


where the function $V_{2}$ is given in the previous section, $V_{3}=-\ln S-\frac{1}{\alpha} \ln Z$ and $V_{4}=\frac{1}{m+1}\left(S+I+R+\frac{\mu+\delta}{2 \alpha} Z\right)^{m+1}+c R . M, m$ and $c$ are positive constants satisfying some conditions as we shall see later.

Since $V_{6}$ is continuous and tends to $\infty$ as $(S, I, R, Z)$ tends to the boundary of $\mathbb{R}_{+}^{4}$, it admits a lower bound $M^{*}$ and achieves this lower bound at a point in $\mathbb{R}_{+}^{4}$. Therefore, we construct the nonnegative function $\tilde{V}_{5}$ by

$$
\tilde{V}_{5}(S, I, R, Z)=V_{5}(S, I, R, Z)-M^{*} .
$$

By simple computations, we can get that

$$
\mathcal{L} V_{2} \leq-\Theta+\beta \beta_{1} I
$$

where

$$
\Theta=\frac{K}{\beta_{1}}\left(1-\frac{1}{\mathcal{R}_{s}^{0}}\right)>0
$$

Moreover,

$$
\mathcal{L} V_{3}=-\frac{\mu}{S}-\frac{I}{Z}+\beta Z+1+\mu+\frac{\sigma_{1}^{2}}{2}+\frac{\sigma_{4}^{2}}{2 \alpha} .
$$

Similarly, we can deduce that

$$
\begin{aligned}
\mathcal{L} V_{4}= & \left(S+I+R+\frac{\mu+\delta}{2 \alpha} Z\right)^{m}\left(\mu-\mu S-\frac{\mu+\delta}{2} I-\mu R-\frac{\mu+\delta}{2} Z\right) \\
& +\frac{m}{2}\left(S+I+R+\frac{\mu+\delta}{2 \alpha} Z\right)^{m-1}\left(\sigma_{1}^{2} S^{2}+\sigma_{2}^{2} I^{2}+\sigma_{3}^{2} R^{2}+\sigma_{4}^{2} Z^{2}\right) \\
& +c \lambda I-c(\mu+\gamma) R .
\end{aligned}
$$

We choose $m$ sufficiently small so that the following inequality holds,

$$
m\left(\sigma_{1}^{2} \vee \sigma_{2}^{2} \vee \sigma_{3}^{2} \vee \sigma_{4}^{2}\right)<\min \left\{\mu, \frac{\mu+\delta}{2}, \frac{(\mu+\delta)^{2}}{4 \alpha}\right\}
$$

Then, we have

$$
\begin{aligned}
\mathcal{L} V_{4} \leq & -\mu m S^{m+1}-\frac{\mu+\delta}{4} I^{m+1}-\frac{\mu}{2} R^{m+1}-c(\mu+\gamma) R \\
& -\frac{1}{2 \alpha^{m}}\left(\frac{\mu+\delta}{2}\right)^{m+1} Z^{m+1}+\Upsilon
\end{aligned}
$$


where

$$
\begin{aligned}
\Upsilon= & \sup _{(S, I, R, Z) \in \mathbb{R}_{+}^{4}}\left\{-\mu(1-m) S^{m+1}-\frac{\mu+\delta}{4} I^{m+1}-\frac{\mu}{2} R^{m+1}\right. \\
& -\frac{1}{2 \alpha^{m}}\left(\frac{\mu+\delta}{2}\right)^{m+1} Z^{m+1}+c \lambda I \\
& +\frac{m}{2}\left(\sigma_{1}^{2} \vee \sigma_{2}^{2} \vee \sigma_{3}^{2} \vee \sigma_{4}^{2}\right)\left(S+I+R+\frac{\mu+\delta}{2 \alpha} Z\right)^{m-1}\left(S^{2}+I^{2}+R^{2}\right) \\
& \left.+\mu\left(S+I+R+\frac{\mu+\delta}{2 \alpha} Z\right)^{m}\right\} .
\end{aligned}
$$

Hence,

$$
\begin{aligned}
\mathcal{L} \tilde{V}_{5} \leq & -M \Theta+\beta \beta_{1} I-c(\mu+\gamma) R-\frac{\mu}{S}-\frac{I}{Z}-\mu m S^{m+1} \\
& -\frac{\mu+\delta}{4} I^{m+1}-\frac{\mu}{2} R^{m+1}-\frac{1}{4 \alpha^{m}}\left(\frac{\mu+\delta}{2}\right)^{m+1} Z^{m+1}+\Psi,
\end{aligned}
$$

where

$$
\Psi=\sup _{Z>0}\left\{-\frac{1}{4 \alpha^{m}}\left(\frac{\mu+\delta}{2}\right)^{m+1} Z^{m+1}+\beta Z+\Upsilon+1+\mu+\frac{\sigma_{1}^{2}}{2}+\frac{\sigma_{4}^{2}}{2 \alpha}\right\} .
$$

Let $\varepsilon_{i}, i=1, \cdots, 3$ be positive reals and define the closed set

$D=\left\{(S, I, R, Z) \in \mathbb{R}_{+}^{4} ; \varepsilon_{1} \leq S \leq \frac{1}{\varepsilon_{1}}, \varepsilon_{2} \leq I \leq \frac{1}{\varepsilon_{2}}, \varepsilon_{2}^{2} \leq S \leq \frac{1}{\varepsilon_{2}^{2}}\right.$, and $\left.\varepsilon_{3} \leq Z \leq \frac{1}{\varepsilon_{3}}\right\}$.

We divide the domaine $\mathbb{R}_{+}^{4} \backslash D$ into eight domains

$$
\begin{aligned}
& D_{1}=\left\{(S, I, R, Z) \in \mathbb{R}_{+}^{4} ; S<\varepsilon_{1}\right\}, \quad D_{2}=\left\{(S, I, R, Z) \in \mathbb{R}_{+}^{4} ; S>\frac{1}{\varepsilon_{1}}\right\}, \\
& D_{3}=\left\{(S, I, R, Z) \in \mathbb{R}_{+}^{4} ; Z<\varepsilon_{3}\right\}, \quad D_{4}=\left\{(S, I, R, Z) \in \mathbb{R}_{+}^{4} ; Z>\frac{1}{\varepsilon_{3}}\right\}, \\
& D_{5}=\left\{(S, I, R, Z) \in \mathbb{R}_{+}^{4} ; S \geq \varepsilon_{1}, I<\varepsilon_{2}, Z \geq \varepsilon_{3}\right\}, \\
& D_{6}=\left\{(S, I, R, Z) \in \mathbb{R}_{+}^{4} ; R>\frac{1}{\varepsilon_{2}^{2}}\right\}, \\
& D_{7}=\left\{(S, I, R, Z) \in \mathbb{R}_{+}^{4} ; I>\varepsilon_{2}, R<\varepsilon_{2}^{2}\right\}, D_{8}=\left\{(S, I, R, Z) \in \mathbb{R}_{+}^{4} ; I>\frac{1}{\varepsilon_{2}}\right\} .
\end{aligned}
$$

Let us show that $\mathcal{L} \tilde{V}_{5}$ is negative at any vector in $\mathbb{R}_{+}^{4} \backslash D$.

Case 1. If $(S, I, R, Z) \in D_{1}$, we use the fact that $-\frac{\mu}{S}<-\frac{\mu}{\varepsilon_{1}}$ and choose $\varepsilon_{1}$ 
sufficiently small such that

$$
\begin{aligned}
\mathcal{L} \tilde{V}_{5} & \leq-M \Theta+\beta \beta_{1} I-\frac{\mu}{S}-\frac{\mu+\delta}{4} I^{m+1}+\Psi \\
& \leq-M \Theta-\frac{\mu}{\varepsilon_{1}}+\Psi_{1} \\
& <0
\end{aligned}
$$

where $\Psi_{1}=\sup _{Z>0}\left\{\beta \beta_{1} I-\frac{\mu+\delta}{4} I^{m+1}+\Psi\right\}$.

Case 2. Let $(S, I, R, Z) \in D_{2}$. Since $-\mu m S^{m+1}<-\frac{\mu m}{\varepsilon_{1}^{m+1}}$, we choose $\varepsilon_{1}$ sufficiently small such that

$$
\begin{aligned}
\mathcal{L} \tilde{V}_{5} & \leq-M \Theta-\mu m S^{m+1}-m u \frac{m}{2} S^{m+1}+\Psi_{1} \\
& \leq-M \Theta-\frac{\mu m}{\varepsilon_{1}^{m+1}}+\Psi_{1} \\
& <0
\end{aligned}
$$

Case 3. If $(S, I, R, Z) \in D_{3}$, then, there exists a sufficiently large $M$ such that

$$
\mathcal{L} \tilde{V}_{5} \leq-M \Theta+\Psi_{1}<0
$$

Case 4. If $(S, I, R, Z) \in D_{4}$. With the fact that $-Z^{m+1}<-\frac{1}{\varepsilon_{3}^{m+1}}$, we choose $\varepsilon_{3}$ sufficiently small such that

$$
\begin{aligned}
\mathcal{L} \tilde{V}_{5} & \leq-M \Theta-\frac{1}{4 \alpha^{m}}\left(\frac{\mu+\delta}{2}\right)^{m+1} Z^{m+1}+\Psi_{1} \\
& \leq-M \Theta-\frac{1}{4 \alpha^{m}}\left(\frac{\mu+\delta}{2 \varepsilon_{3}}\right)^{m+1}+\Psi_{1}
\end{aligned}
$$

$<0$.

Case 5. If $(S, I, R, Z) \in D_{5}$, there is a sufficiently large number $M$ such that

$$
\mathcal{L} \tilde{V}_{5} \leq-M \Theta+\beta \beta_{1} \varepsilon_{2}+\Psi_{1}<0
$$

Case 6. Similarly to the third case, if $(S, I, R, Z) \in D_{5}$, with a large value of $M$ one can get

$$
\mathcal{L} \tilde{V}_{5} \leq-M \Theta+\Psi_{1}<0
$$


Case 7. If $(S, I, R, Z) \in D_{7} \cup D_{8}$, there is a sufficiently large number $M$ such that

$$
\mathcal{L} \tilde{V}_{5} \leq-M \Theta+\Psi_{1}<0
$$

With the above choices of $M, \varepsilon_{1}$ and $\varepsilon_{3}$ and for a given $\varepsilon_{2}<1$, we get

$$
\mathcal{L} \tilde{V}_{5}(S, I, R, Z)<0, \quad \text { for any } \quad(S, I, R, Z) \in \mathbb{R}_{+}^{4} \backslash D .
$$

This concludes the proof.

\section{Numerical simulations and conclusion}

Adopting Milstein's scheme for SDE discretization [10, we simulated system (3) for many parameter sets with the aim of illustrating the results exhibited in this paper. We choose the initial value $(S(0), I(0), R(0))=(0.5,0.15,0.1)$ and use R software to perform simulations. Theorem 3.1 presents conditions under

Table 1: Parameters values used in Fig. 1

\begin{tabular}{cccccccccc}
\hline$\mu$ & $\beta$ & $\lambda$ & $\delta$ & $\gamma$ & $\alpha$ & $\sigma_{1}$ & $\sigma_{2}$ & $\sigma_{3}$ & $\sigma_{4}$ \\
0.12 & 0.27 & 0.48 & 0.1 & 0.113 & 0.11 & 0.2 & 0.23 & 0.15 & 0.07 \\
\hline
\end{tabular}

Table 2: Parameters values used in Fig. 2 and Fig. 3

\begin{tabular}{cccccccccc}
\hline$\mu$ & $\beta$ & $\lambda$ & $\delta$ & $\gamma$ & $\alpha$ & $\sigma_{1}$ & $\sigma_{2}$ & $\sigma_{3}$ & $\sigma_{4}$ \\
0.09 & 0.37 & 0.328 & 0.1 & 0.11 & 0.07 & 0.05 & 0.04 & 0.035 & 0.04 \\
\hline
\end{tabular}

which the disease dies out exponentially. This is illustrated by Fig. 1 where sufficient conditions are satisfied.

$$
\begin{gathered}
\mathcal{R}_{0}=0.7183<1, \quad \sigma_{1}^{2}=0.04 \leq 2 \mu=0.24 \quad \text { and } \\
\min \{\mu+\lambda+\delta, \mu+\gamma, \alpha\}\left(\sqrt{\mathcal{R}_{0}}-1\right)+\frac{\beta \alpha \sigma_{1} \sqrt{\mathcal{R}_{0}}}{(\mu+\lambda+\delta) \sqrt{2 \mu-\sigma_{1}^{2}}}=-7 \cdot 10^{-4}<0 .
\end{gathered}
$$



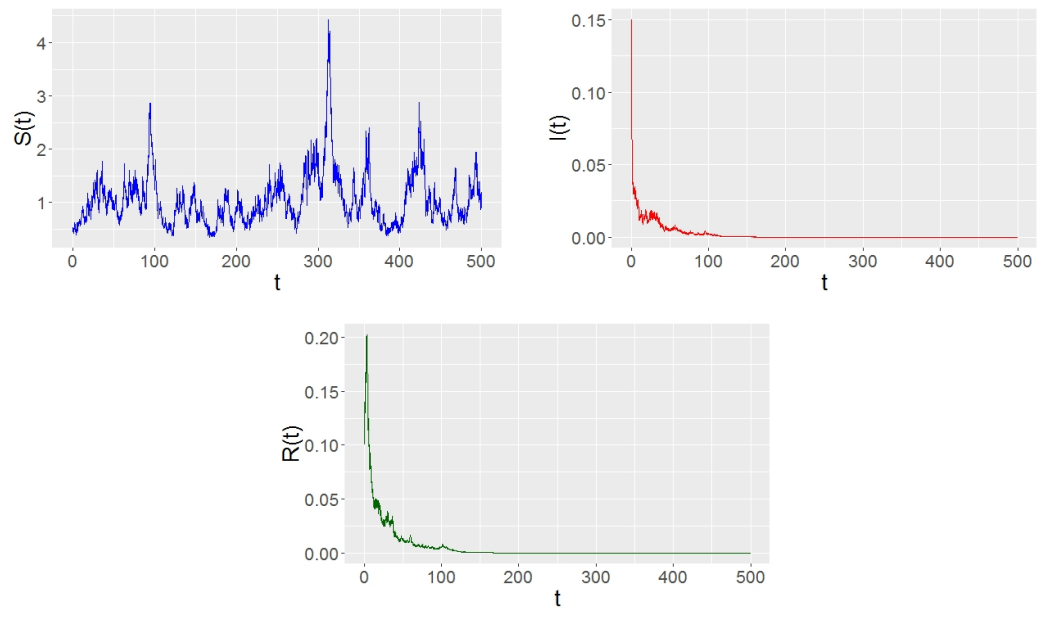

Figure 1: Trajectories of $S(t), I(t)$ and $R(t)$ components of $(S(t), I(t), R(t), Z(t))$ the solution of system 3 with parameters given in Table 1

40 When $\mathcal{R}_{s}^{0}$ exceeds 1 , the disease should be prevalent with probability one. This confirms the behaviour of the epidemic in Fig. 2 with parameters given in table 2 and a value of $\mathcal{R}_{s}^{0}$ equals to 1.0636.

By sampling 10000 stochastic paths of the solution to system (3) and keeping the same parameter set presented in table 2 , the stationary probability density

45 functions of infected and removed individuals at different times are shown in Fig. 3 respectively. We see that they are close to each other at $t=2000$ and $t=3000$, which is confirmed by theorem 5.1 .

To conclude, a stochastic SIRI epidemic model with distributed delay is analyzed in this paper. The existence and uniqueness of a global positive solution

${ }_{50}$ is the first result we established. Some sufficient conditions are obtained to promise the extinction of the disease. $\mathcal{R}_{s}^{0}>0$ is an adequate condition to the permanence of the disease as well as the existence of unique stationary distribution to our model. As an important question, it will be noteworthy to study the behaviour of the considered model in the case where $\mathcal{R}_{0} \leq 1 \leq \mathcal{R}_{s}^{0}$. We leave ${ }_{55}$ the response to this question to further works. 

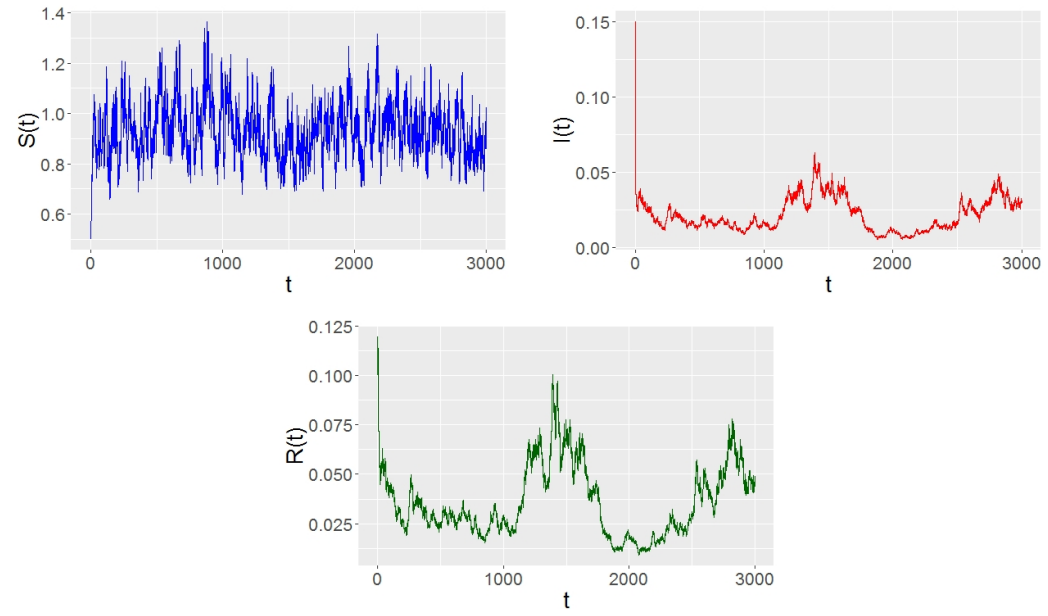

Figure 2: Simulation of paths $S(t), I(t)$ and $R(t)$ where $(S(t), I(t), R(t), Z(t))$ is the solution to system 3 using data of Table 2
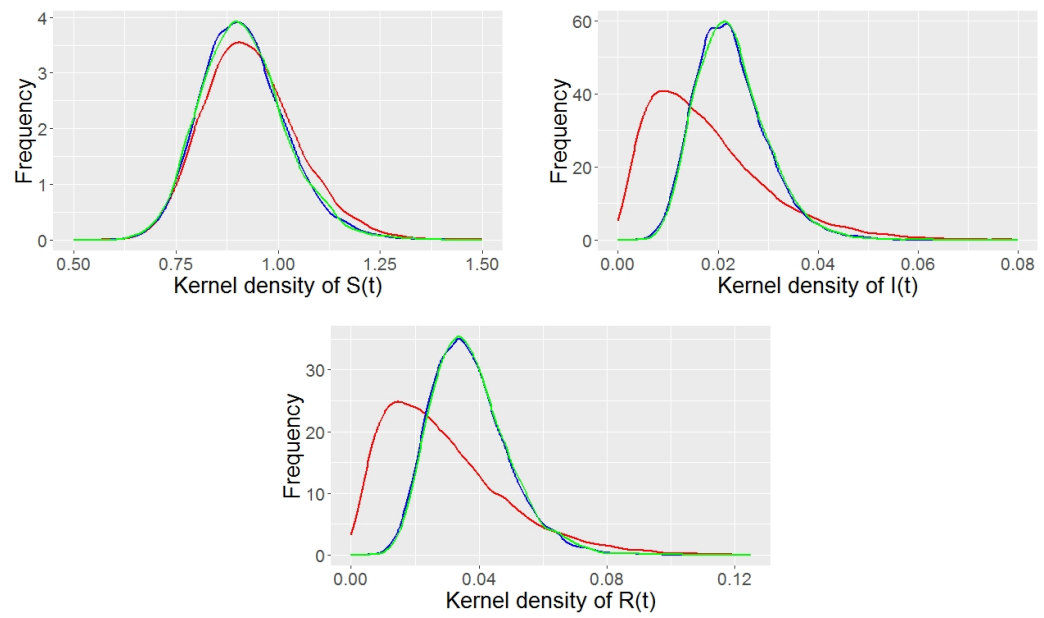

Figure 3: The kernel density functions of susceptible, infected and removed compartments of (3) at $t=1000$ (red), $t=2000$ (blue) and $t=3000$ (green).

Acknowledgements. The research of T. Caraballo has been partially supported by Ministerio de Ciencia, Innovación y Universidades (Spain), FEDER (European Community) under grant PGC2018-096540-B-I00. 


\section{References}

[1] B.-E. Berrhazi, M. El Fatini, T. Caraballo, R. Pettersson, A stochastic SIRI epidemic model with Lévy noise. Discrete Contin. Dyn. Syst. Ser. B 23 (6) (2018), 2415-2431.

[2] B.-E. Berrhazi, M. El Fatini, R. Pettersson, A. Laaribi, Media effects on the dynamics of a stochastic SIRI epidemic model with relapse and Lévy noise perturbation. Int. J. Biomath. 12 (3) (2019) 1950037, 21 pp.

[3] T. Caraballo, R. Colucci, A comparison between random and stochastic modeling for a SIR model. Commun. Pure Appl. Anal. 16 (2017), no. 1, 151-162.

[4] T. Caraballo, M. El Fatini, R. Pettersson, R. Taki, A stochastic SIRI epidemic model with relapse and media coverage. Discrete Contin. Dyn. Syst. Ser. B 23 (8) (2018), 3483-3501.

[5] M. El Fatini, B. Boukanjime, Stochastic analysis of a two delayed epidemic model incorporating Lévy processes with a general non-linear transmission, Stoch. Anal. Appl. (2019) doi: 10.1080/07362994.2019.1680295.

75 [6] M. El Fatini, M. El Khalifi, R. Gerlach, A. Laaribi, R. Taki, Stationary distribution and threshold dynamics of a stochastics SIRS model with a general incidence, Physica A 534 (2019) 120696.

[7] M. El Fatini, A. Laaribi, R. Pettersson, R. Taki, Lévy noise perturbation for an epidemic model with impact of media coverage. Stochastics 91 (7) (2019),998-1019.

[8] M. El Fatini, A. Lahrouz, R. Pettersson, A. Settati and R. Taki, Stochastic stability and instability of an epidemic model with relapse, Appl. Math. Comput. 316 (2018) 326-341.

[9] M. El Fatini, I. Sekkak, A. Laaribi, A threshold of a delayed stochastic epidemic model with Crowly-Martin functional response and vaccination, Physica A 520 (2019) 151-160. 
[10] D. J. Higham, An Algorithmic introduction to numerical simulation of stochastic differential equations, SIAM Rev. 43 (2001) 525-546.

[11] Institute for Health Metrics and Evaluation (IHME), Findings from the Global Burden of Disease Study 2017. Seattle, WA: IHME, 2018.

[12] C. Ji, D. Jiang, Q. Yang, N. Shi, Dynamics of a multigroup SIR epidemic model with stochastic perturbation, Automatica 48 (1) (2012) 121-131.

[13] W. O. Kermack, A.G. McKendrick, A contribution to the mathematical theory of epidemics, Proc. R. Soc. Lond. A, 115 (1927) 700-721.

${ }_{95}[14]$ W. O. Kermack, A.G. McKendrick, Contributions to the mathematical theory of epidemics, part. II. Proc. R. Soc. Lond. A, 138 (1932) 55-83.

[15] W. O. Kermack, A.G. McKendrick, Contributions to the mathematical theory of epidemics, part. III. Proc. R. Soc. Lond. A, 141 (1933) 94-112.

[16] R. Khasminskii, Stochastic Stability of Differential Equations, Springer, 2012.

[17] A. Y. Kutoyants, Statistical Inference for Ergodic Diffusion Processes, Springer, London 2003.

[18] A. Lahrouz, A. Settati, M. El Fatini, R. Pettersson, R. Taki, Probability analysis of a perturbed epidemic system with relapse and cure, International Journal of Computational Methods 16, 2018. doi:10.1142/s0219876218501402.

[19] Q. Liu, D. Jiang, T. Hayat, A. Alsaedi, Dynamics of a stochastic SIR epidemic model with distributed delay and degenerate diffusion, J. Franklin Inst. 356 (13) (2019) 7347-7370.

${ }_{110}$ [20] Q. Lu, Stability of sirs system with random perturbations, Physica A 388 (18) (2009) 3677-3686. 
[21] W. Ma, Y. Takeuchi, T. Hara, E. Beretta, Permanence of an SIR epidemic model with distributed time delays, Tohoku Math. J. 54 (2002) 581-591.

[22] N. Macdonald, Time Lags in Biological Models, Lecture Notes in Biomathematics, Springer-Verlag, Heidelberg, 1978.

[23] H. Shu, D. Fan, J. Wei, Global stability of multi-group SEIR epidemic models with distributed delays and nonlinear transmission, Nonlinear Anal.: RWA 13 (2012) 1581-1592.

[24] E. Tornatore, S. Buccellato, P. Vetro, Stability of a stochastic sir system, Physica A 354 (C) (2005) 111-126.

[25] C. Xu, X. Li, The threshold of a stochastic delayed SIRS epidemic model with temporary immunity and vaccination, Chaos Solitons Fract. 111 (2018) 227-234.

[26] J. Yu, D. Jiang, N. Shi, Global stability of two-group sir model with random perturbation, J. Math. Anal. Appl. 360 (2009) 235-244.

[27] T. Zhang, X. Meng, T. Zhang, Y. Song, Global dynamics for a new highdimensional SIR model with distributed delay, Appl. Math. Comput. 218 (2012) 11806-11819.

[28] Y. Zhao and D. Jiang. The threshold of a stochastic sirs epidemic model with saturated incidence, Appl. Math. Lett. 34 (2014) 90-93.

[29] C. Zhu and G. Yin. Asymptotic properties of hybrid diffusion systems, SIAM J. Control Optim. 46 (4) (2007) 1155-1179. 